\title{
A review on hormonal complications of gastrointestinal system and its treatment during prenatal period
}

\author{
Dr. A. V. Kishore Babu, Dr. A. Srinivasa Rao \\ Correspondence: Dr. A V Kishore Babu, Bhaskar Pharmacy College, Hyderabad, Telangana, \\ India; Email- kishorebabu.pharma@gmail.com
}

Distributed under Creative Commons Attribution-Share Alike 4.0 International.

\begin{abstract}
The endocrine system of human pregnancy involves hormonal and metabolic changes that result from physiological alterations at the boundary between mother and fetus. Initially, the ovaries, and then later, the placenta, are the main producers of pregnancy-related hormones that are essential in creating and maintaining the correct conditions required for a successful pregnancy. Progesterone and estrogen have a great role, receptors for these hormones have been found on gastrointestinal cells, which suggest that the gastrointestinal tract is designed to sense and react to them. The altered levels of these hormones changes normal physiological functions of gastrointestinal tract and leads to morning sickness (nausea and vomiting), acid reflux, heartburn, indigestion, abdominal bloating, diarrhea and constipation. The goal of treatment is to improve symptoms while minimizing risks to mother and fetus. To attain this, a multimodal approach tailored to each individual is usually needed. Treatment modalities range from simple dietary modifications to drug therapy and total parental nutrition.
\end{abstract}

Keywords: Pregnancy, gastrointestinal system, human chorionic gonadotropin, estrogen, morning sickness, heartburn and constipation.

Pregnancy is supposed to be one of the happiest times of a woman's life. In general female life cycle is associated with a number of hormonal milestones include: menarche, pregnancy, contraceptive use, menopause, and the use of replacement sex hormones. Menarche marks the onset of menses and cyclic changes in hormone levels. Pregnancy is associated with rising non cyclic levels of sex hormones, and menopause with declining non cyclic levels. Hormonal contraceptive use during the reproductive years and hormone replacement in menopause are therapeutic hormonal interventions that alter the levels and cycling of sex hormones [1]. At the same time hormonal and physiologic changes during pregnancy are unique for pregnant women. The endocrine system of human pregnancy involves hormonal and metabolic changes that result from physiological alterations at the boundary between

Received: $16^{\text {th }}$ June 2016. Accepted: $5^{\text {th }}$ September 2016.

Kishore Babu AV, Rao AS. A review on hormonal complications of gastrointestinal system and its treatment during prenatal period. The New Indian Journal of OBGYN. 2017; 3(2): 70-8 
mother and fetus [2]. The correct balance of hormones is essential for a successful pregnancy. Initially, the ovaries, and then later, the placenta, are the main producers of pregnancy-related hormones that are essential in creating and maintaining the correct conditions required for a successful pregnancy. Progesterone and estrogen have a great role along with other hormones like human chorionic gonadotropin (HCG), human placental lactogen (HPL), prolactin, corticotrophin-releasing hormone, oxytocin, relaxin, inhibin, cortisol and aldosterone [2,3]. Gastrointestinal symptoms are extremely common during pregnancy. Increased levels of hormones contribute to symptoms such as nausea, vomiting, heartburn, acid reflux, abdominal bloating and constipation.

The goal of treatment is to improve symptoms while minimizing risks to mother and fetus. To attain this, a multimodal approach tailored to each individual is usually needed. Treatment modalities range from simple dietary modifications to drug therapy and total parental nutrition. Severity of symptoms and maternal weight loss are useful in determining the aggressiveness of treatment.

\section{1) Nausea and Vomiting in pregnancy (NVP)}

It is estimated that $70-80 \%$ of pregnant women experience NVP [4]. NVP is found more often in Western countries and urban populations and is rare among Africans, Native Americans, Eskimos, and most Asian populations [5]. Most of the women will experience nausea and vomiting during the first trimester than later stages of pregnancy and varies in severity from mild to hyperemesis gravidarum [6-8]. Unfortunately, it isn't clear what causes nausea and vomiting. Various explanations have been put forward but the most implicated factor is human chorionic gonadotropin (HCG), lack of vitamin B6 and disturbances in gastric motility have a greater role in the pathophysiology of nausea and vomiting. Higher urinary hCG [5] and serum HCG levels have also been found in women with NVP compared to those who are asymptomatic [9]. Estrogen is another suggested cause with the presence of a female fetus reported to increase the likelihood of severe nausea and vomiting during pregnancy [10]. Progesterone in combination with estrogen may also have a role in NVP. Progesterone decreases smooth muscle contractility and may alter gastric emptying and lead to increased nausea and vomiting [11]. In some instance several other factors also contribute to NVP including placental prostaglandin E2 (PGE2) [12], pregnancy-associated alterations to the vestibular system, taste and olfaction, and behavioral and psychological aspects [7,13].

When limited to the gastrointestinal tract, gastric dysrhythmia leads to stomach dysfunction and nausea in pregnancy $[7,14]$. Gastric dysrhythmias are probably caused by increased levels of female sex hormones, but raised levels of vasopressin might also have a role [7,8]. Moreover, increased release of nitric oxide and down regulation of stimulatory G-protein expression (which stimulate muscle contraction) and up regulation of inhibitory G-protein expression have been detected in gastric and intestinal muscle preparations from pregnant animals [15-17]. These changes could explain how gastric contractility is reduced and consequently how gastric emptying is impaired. Recurrent abdominal pain can be caused by gastrointestinal dysmotility and can occur in patients with nausea and vomiting of pregnancy; however, such pain can also be a symptom of other gastrointestinal diseases (e.g. GERD, ulcers, cholecystitis, and pancreatitis) that need to be excluded as appropriate, depending on the individual patient's presenting symptoms and signs [7].

Adverse outcomes, however, have been reported in some studies, especially when NVP is deemed severe. In a study, Zhou et al found an increased risk for low birth weight in women with severe NVP, likely due to the deleterious effects of nausea and vomiting on maternal nutrition [18].

\section{Treatment}

\section{A) Nonpharmacological Treatment}

Reassurance and the avoidance of precipitating factors are the first therapeutic steps to be recommended in patients with mild symptoms.

Dietary measures - The diet should consist of frequent, several small meals that are high in proteins, carbohydrate, taking more liquids than solids and low 
in fat (fatty foods may further delay gastric emptying) throughout the day may help nausea by improving the gastric dysrhythmias associated with NVP [19]. Small volumes of salty liquids such as electrolytesreplacement sport beverages are advisable, and if the smell of hot foods is noxious, cold foods should be prepared [20].

Emotional support - Emotional support should always be offered by a medical professional. In addition, supportive psychotherapy, behavioral therapy, and hypnotherapy may be beneficial to women with severe symptoms and/or those in whom personality characteristics, marital or family conflict play a role [21]. The goal of psychotherapy is not to delve into the psychology which may be contributing to NVP but rather to encourage, explain, reassure, and allow the patient to express stress [22].

Acupressure/acupuncture - Acupressure of the Chinese acupuncture point P6 (Neiguan), which is found on the inside of the forearm three fingerbreadths above the wrist has been found to decrease nausea in patients with chemotherapy-induced nausea and postoperative nausea and vomiting and may be helpful in treating HG. According to the principle of chi, application of pressure to this point blocks abnormal energy slow and relives symptoms related to the pressure point [23]. Moreover, stimulation of the P6 acupuncture point, applied by finger pressure, a wristband, or the ReliefBand, a battery-operated electrical nerve stimulator worn on the wrist has recently been approved by the FDA and can also be used to stimulate the P6 site [24]. While studies regarding the benefits of acupressure have been inconclusive, some experts believe this intervention should be offered as there are no known adverse side effects [25].

Ginger - Ginger is the single nonpharmacologic intervention recommended by the American College of Obstetrics and Gynecology [26]. Evidence also suggests that ginger (Zingiber officinale) might be an effective treatment for relieving the severity of nausea and decreasing the number of vomiting episodes in pregnancy [27]. Ginger is believed to help improve
NVP by stimulating gastrointestinal tract motility and stimulating the flow of saliva, bile, and gastric secretions. One component of ginger has been shown to have similar activity as the 5-HT3 antagonist like ondansetron [28]. A trial of 70 pregnant women at 17 weeks' gestation or less treated with either $250 \mathrm{mg}$ of ginger four times a day or a placebo for 4 days found that women in the treatment group had significant improvement in nausea symptoms compared with women in the placebo group $(\mathrm{P}<.001)$ [29].

\section{B) Pharmacologic Treatment}

Pyridoxine (vitamin B6, FDA category A) - It is used first-line in many countries for nausea and vomiting in early pregnancy, however, there are large individual differences in its onset and action [30]. Studies have shown that pyridoxine improves mild to moderate nausea but does not significantly reduce vomiting [31].

Antihistamines - Antihistamines indirectly affect the vestibular system, decreasing stimulation of the vomiting center.

Doxylamine (FDA category B) - Doxylamine is an H1 receptor antagonist that has been shown to be effective in the treatment of NVP. The combination of pyridoxine (vitamin B6) (pregnancy category A) and doxylamine (category B), is the only medication that is specifically labeled for the treatment of NVP by the Food and Drug Administration.

Other Antihistamines - Other H1 receptor antagonists like dimenhydrinate, diphenhydramine, meclizine, cyclizine and hydroxyzine are considered safe in pregnancy, with no human teratogenic potential. A meta-analysis of more than 24 controlled studies with more than 200,000 pregnant women found that antihistamines (H1 blockers, in particular) given during the first trimester did not increase teratogenic risk [32]. While meclizine had been previously thought to be teratogenic, studies now show it is safe to use during pregnancy [33]. Dimenhydrinate and diphenhydramine have conflicting results on safety [34].

Trimethobenzamide - Trimethobenzamide is a pregnancy category $\mathrm{C}$ drug. There are 3 studies that have followed up outcomes in women who used 
trimethobenzamide in their first trimester for nausea and vomiting, and there was no increase in the incidence of malformations in all 3 studies [35]. There are no data regarding the use of this agent during breast-feeding.

Phenothiazines - Like antihistamines, phenothiazines like chlorpromazine, perphenazine, prochlorperazine, promethazine, trifluoperazine have also been proven safe for use in pregnancy. Prospective and retrospective cohort, case-control, and record-linkage studies of patients with exposure to various and multiple phenothiazines have failed to demonstrate an increased risk for major malformations [35].

Metoclopramide - It is one of the most commonly prescribed medicines NVP. A Danish study identified 309 women over a 5-year period with singleton pregnancies and prescriptions for metoclopramide. As compared with 13,327 controls, there were no major differences in the risk for malformations (OR, 1.11; 95\% CI, $0.6-2.1$ ), low birth weight (OR, 1.79; 95\% CI, $0.8-3.9$ ), or preterm delivery (OR, $1.02 ; 95 \% \mathrm{CI}, 0.6-$ 1.7) [36]. Despite its efficacy, metoclopramide use is limited by its side effect profile which includes dystonia, restlessness, and somnolence. In 2009 the FDA added a black box warning to metoclopramide due to the risk of tardive dyskinesia with chronic use.

Domperidone (FDA category C) - It is a dopamine antagonist and prokinetic agent used for short-term treatment of nausea and vomiting but its usage is not been studied in NVP. However, droperidol is another dopamine antagonist bears a black box warning as it may cause QT prolongation and cardiac dysrhythmias.

Corticosteroids - Corticosteroids are pregnancy category C drugs. A case-control study of corticosteroid use during the first trimester of pregnancy noted an increased risk of oral clefts in the newborn [37]. A prospective controlled study of 311 women who received glucocorticosteroids during the first trimester did not note an increased rate of major anomalies and no cases of oral cleft were noted [38]. Overall, the use of corticosteroids poses a small risk to the developing infant and the mother needs to be informed of both the benefits and the risks of therapy.
Prednisone and Prednisolone are compatible with breast-feeding.

Ondansetron (FDA category B) - It is a 5-HT3 antagonist, is widely used for the treatment of postoperative and chemotherapy-induced nausea and vomiting and is currently one of the most commonly prescribed anti-emetics [39]. Its safety in pregnancy was determined in a recent study which showed no significant increase in the number of miscarriages, major malformations or birth weight between infants exposed to ondansetron and unexposed controls [40].

Granisetron and Dolasetron - Granisetron and dolasetron are both pregnancy category B drugs. There have been no studies on pregnant humans exposed to these agents. However, pregnant rats and rabbits administered doses up to 146 times those used in humans have failed to demonstrate any adverse outcomes. Similarly, there are no data regarding their safety in breast-feeding.

\section{2) Acid reflux and heartburn}

Acid reflux and heartburn are the normal consequencess in pregnancy with GERD. "Heartburn" is sometimes used interchangeably with "acid reflux." Acid reflux is when stomach acid splashes up from the stomach into the esophagus and they are associated with gastrointestinal dysmotility. Gastroesophageal reflux disease (GERD) is reported in up to $80 \%$ of pregnancies [41]. The predominant etiologies are alterations in lower esophageal sphincter (LES) resting pressure and esophageal peristalsis. Generally in the first trimester of pregnancy, basal lower oesophageal sphincter (LES) pressure may not change, but is less responsive to physiological stimuli (i.e. pentagastrin, edrophonium chloride, methacholine or a protein meal) that usually increase LES pressure [42]. In the later two trimesters, LES pressure gradually falls approximately $33-50 \%$ of basal values reaching a nadir at 36 weeks of gestation and rebounds to prepregnancy values 1-4 weeks postpartum [43]. Animal and human studies find that estrogen and progesterone are the likely mediators of esophageal dysmotility in pregnancy wherein estrogen serves as a primer and progesterone causes LES relaxation. 


\section{Treatment}

A) Non pharmacological Treatment: Treatment algorithms suggest stepwise progression of options, starting with lifestyle modifications like eating smaller and more frequent meals, avoid eating near bedtime, and elevate the head of the bed. Trying pharmacologic therapy if symptoms are not adequately managed by lifestyle changes. Chewing gum stimulates the salivary glands and can help neutralize acid. Abstinence from alcohol and tobacco are encouraged to reduce reflux symptoms and to avoid fetal exposure to these harmful substances.

B) Pharmacological treatment: Antacids or sucralfate are considered the first-line drug therapy. If symptoms persist, any of the histamine 2-receptor antagonists can be used. Proton pump inhibitors are reserved for women with intractable symptoms or complicated reflux disease.

Antacids: Only limited data exist concerning the effects of antacids on the fetus with no controlled trials of efficacy. Antacids containing aluminum, calcium, and magnesium were not found to be teratogenic in animal studies and are recommended as first-line treatment of heartburn and acid reflux during pregnancy [44], although $15-30 \%$ of magnesium and a smaller percentage of aluminium preparations are absorbed after reacting with hydrochloric acid. A recent European consensus conference recommended calcium/magnesium- based antacids for pregnant women because of their safety profile [45]. These experts found that calcium based antacids had the added benefit of increasing calcium supplementation to prevent the hypertension and pre-eclampsia associated with pregnancy. In addition, a large, randomized placebo-controlled trial found that magnesium sulphate supplementation reduces the risk of eclampsia by $50 \%$ compared with placebo, and may also reduce the risk of maternal death, with no serious short-term side-effects [46]. However, 10 adverse events were reported in 10 fetuses (three episodes of fetal distress) and others report that high-dose and prolonged use of Gaviscon compounds containing magnesium trisilicate is associated with nephrolithiasis, hypotonia, and respiratory distress in the fetus, and its use is not recommended during pregnancy [47]. Bicarbonatecontaining antacids are also not recommended owing to the risk of maternal and fetal metabolic acidosis and fluid overload.

Metoclopramide: It is FDA category B drug during pregnancy. Animal studies with doses up to 250 times the recommended human dose reveal no evidence of impaired fertility or fetal toxicity. In the Michigan Medicaid Surveillance Study, 10 (5.2\%) major birth defects were reported in 992 newborns exposed to metoclopramide during the first trimester, but congenital malformations or fetal toxicity have not been reported in humans [48].

Cisapride: It is FDA category $\mathrm{C}$ drug in pregnancy because of its toxicity in animals. Human reports suggest cisapride is safe during pregnancy. In a prospective, multicentre study, the outcome of 129 Canadian women who took cisapride during pregnancy between November 1996 and November 1998 were compared with a control group. Most women took cisapride during the first trimester $(88 \%), 3 \%$ of women took it throughout their pregnancy. Investigators found no differences in rates of major or minor congenital malformations in the cisapride group compared with the matched controls [49].

Histamine-2 receptor antagonists (H2RAs): The H2RAs are the most commonly used and safest medications for the pregnant woman who were not responding to lifestyle modification and nonabsorbable medication. Drugs like cimetidine, ranitidine, famotidine and nizatidine are FDA approved category $\mathrm{B}$ drugs for pregnancy. Over the last 30 years cimetidine and ranitidine have considerable use in pregnancy with an excellent safety profile. In animal studies, cimetidine has a weak antiandrogenic effect in animals, as evidenced by a reduction of the size of testes, prostate glands and seminal vesicles [50]. Ranitidine has no antiandrogenic activity in animals [51]. Neither H2RA has reports of human sexual defects in infants. In a double-blind, placebocontrolled, triple-crossover study, Larson et al. compared ranitidine once or twice daily with placebo in 
pregnant heartburn subjects not responding to antacids and lifestyle modification. No adverse pregnancy outcomes or drug reactions were noted. Animal studies with famotidine revealed no fetal toxicity or teratogenicity [52]. With nizatidine there is only a single case report of a woman delivering a healthy baby after taking the drug during 14-16 weeks of gestation [48].

Proton pump inhibitors (PPIs): Proton-pump inhibitors are the most effective drug therapy for symptom control and healing of oesophagitis. The PPIs have not been as extensively used in pregnancy as the H2RAs, or is their efficacy proven in pregnancy, and the data about total safety are more limited. Proton pump inhibitors approved by Health Canada include omeprazole, pantoprazole, lansoprazole, esomeprazole, and rabeprazole. All the PPIs are categorized as class B drugs except omeprazole, which categorized as a class $\mathrm{C}$ drug by the FDA because of fetal toxicity. The FDA has received reports of at least 12 birth defects in pregnant women exposed to omeprazole, including anencephaly and hydroencephaly [48]. A recent metaanalysis assessed the risks of congenital fetal malformations in women using PPIs in the first trimester of pregnancy [53]. A total of 593 infants were exposed to PPIs, most (534) received omeprazole. The summary relative risk for all major malformations among any PPI exposure was 1.18 (95\% CI: $0.72-$ 1.94), a non-significant relative risk.

\section{3) Constipation}

It has been estimated that approximately $11 \%$ to $38 \%$ of pregnant women experience constipation [54]. However, the GI responses to estrogen and progesterone slows the automatic waves of muscular contractions in the intestine with increased bowel transit time and pressure from the enlarging uterus are considered major physiologic factors. Other causes include decreased maternal activity, decreased levels of motilin, increased colonic sodium and water absorption, which causes stool to dry out and increased vitamin supplementation (eg, iron and calcium) can further contribute to constipation in the pregnant population. Later in pregnancy, an enlarging uterus might slow onward movement of feces [55]. It is important to note that constipation negatively affects patients' daily lives and is second only to nausea as the most common gastrointestinal complaint in pregnancy.

\section{Treatment}

A) Non pharmacological Treatment: Many patients find relief from constipation with the first line measurements includes; i) Drinking plenty of water, ii) Include plenty of fiber diet, iii) Exercise regularly to keep muscles toned up.

B) Pharmacological Treatment: If the pregnant women's are not getting benefit from the first line measurements, laxatives are the alternative second choice in treating constipation [56]. In general, there are insufficient data on the use of laxatives in pregnancy; however, limited studies have been performed for specific laxatives, and the safety of others can be inferred from information about their systemic absorption [57].

Psyllium and bran, are bulk-forming agents, not absorbed or associated with increased risk of malformations [57]; therefore, they are considered safe for long-term use during pregnancy. However, they are not always effective and might be associated with unpleasant side effects such as gas, bloating, and cramping. Docusate sodium or calcium are stool softeners has not been associated with adverse effects in pregnancy in a number of studies, and it is thus also considered safe to use [57]. There is one case report of maternal chronic use of docusate sodium throughout pregnancy, which was associated with symptomatic hypomagnesaemia in the neonate [58]. Lactulose and polyethylene glycol are osmotic laxatives, that are poorly absorbed systemically [59]. Their use has not been associated with adverse effects; however, individuals might experience side effects such as flatulence and bloating.

\section{4) Diarrhea}

Many women experience diarrhea or frequent watery bowel movements, often within a few hours of taking a meal, during various stages of pregnancy, occurring along with nausea and vomiting and food cravings or aversions. Diarrhea can occur due to 
certain hormonal changes, estrogen and progesterone that take place within the body during this time. There is a common misconception that diarrhea or constipation during pregnancy can cause miscarriage, but in truth, it is perfectly normal to have this symptom, especially during the earlier trimesters. Some women also report having diarrhea before labor.

One or more of the following factors may contribute to cause the problem: i) Changes in the diet to provide the fetus with all the necessary nutrients, ii) The extra water intake for keeping the body hydrated, iii) Pregnancy workouts that improve the intestinal functions and sleep patterns, iv) The iron content in prenatal vitamins, v) Food poisoning, vi) Stomach flu (caused by certain bacteria or viruses), vii) Maternal gastrointestinal conditions like inflammatory bowel disease and irritable bowel syndrome (chronic diarrhea).

\section{Treatment}

Although, it is a common symptom of pregnancy, one should not neglect constant diarrhea as it is one of the most common causes of preterm labor.

\section{A) Non Pharmacological Treatment}

Diarrhea occurring due to changes in the diet or a new pregnancy workout schedule generally subsides automatically after a couple of days. In such instances, the following natural remedies help to retain water and electrolytes, preventing dehydration and providing relief from the symptoms. However, they are insufficient for curing any serious underlying conditions causing the diarrhea. BRAT diet (Banana, Rice, Applesauce, Toast) is commonly recommended. One should opt for whole wheat toast (without butter) and brown rice for better results. Additionally, it is advisable to eat starchy foods to provide the body with vitamins and minerals essential during this time.

\section{B) Pharmacological Treatment}

i) Imodium (FDA pregnancy category B; to be used only for 24 hours after 12 weeks of gestation), ii) Polycarbophil (FDA pregnancy category C), iii) Kaopectate (kaolin-and-pectin), iv)Parepectolin (not classified by FDA; not recommended unless benefits outweigh potential risks).

\section{Conclusion}

Increased levels of female sex hormones during prenatal period of pregnancy cause alterations of gastrointestinal motility, and this concept explains, why gastrointestinal symptoms such as, nausea, vomiting, heartburn, diarrhea and constipation are extremely more common during pregnancy. So the main risk factors of these symptoms need to be excluded in severe cases and in patients with alarm symptoms. On the other hand, avoidance of precipitating factors and dietary changes are the first line therapeutic steps to be recommended in patients with mild symptoms. If symptoms do not respond adequately, drug therapy is often warranted to improve quality of life and to prevent complications.

\section{Conflict of interest: None. Disclaimer: Nil. References}

1. Silberstein S, Merriam G. Sex hormones and headache. Neurology. 1999; 53: S3-13.

2. Kumar $P$, Magon N. Hormones in pregnancy. Niger Med J. 2012; 53(4): 179-83.

3. Gerard J, Tortora, Derrickson B. Principles of Anatomy and Physiology. 12 ed. 2009; pg 1113.

4. O'Brien B, Zhou Q. Variables related to nausea and vomiting during pregnancy. Birth. 1995; 22: 93-100. [PubMed: 7779229].

5. Schoeneck FJ. Gonadotropin hormone concentrations in hyperemesis gravidarum. Am J Obstet Gynecol. 1943; 43:308.

6. Baron TH. Gastrointestinal motility disorders during pregnancy. Ann Intern Med. 1993; 118: 366-75.

7. Koch KL. Gastrointestinal factors in nausea and vomiting of pregnancy. Am J Obstet Gynecol. 2002; 186 (Suppl): S198-S203

8. Goodwin TM. Nausea and vomiting of pregnancy: an obstetric syndrome. Am J Obstet Gynecol. 2002; 186 (Suppl): S184-S189

9. Masson GM, Anthony F, Chau E. Serum chorionic gonadotropin (hCG), schwangerschafts protein1 (SP1), progesterone, and oestradiol levels in patients with nausea and vomiting in early pregnancy. Br J Obstet Gynaecol. 1985; 92:211-15. [PubMed: 3872132] 
10. Sheehan P. Hyperemesis gravidarum: assessment and management. Aust Fam Physician. 2007; 36(9): 698-701.

11. Walsh JW, Hasler WL, Nugent CE, et al. Progesterone and estrogen are potential mediators of gastric slow wave dysrhythmias in nausea of pregnancy. Am J Physiol. 1996; 270:506-14.

12. Sanders KM, Bauer AJ, Publicover NG. Regulation of gastric antral slow wave frequency by prostaglandins. In: Roman C, editor. Gastrointestinal motility. Lancaster: MTP Press; 1983. p.77-85.

13. Kenneth L, Koch MD. Gastrointestinal factors in nausea and vomiting of pregnancy. Am J Obstet Gynecol. 2002;186: S198-203.

14. Leahy A, Besherdas K, Clayman C, Mason I, Epstein O. Gastric dysrhythmias occur in gastro-oesophageal reflux disease complicated by food regurgitation but not in uncomplicated reflux, Gut. 2001;48:212-15.

15. Shah $\mathrm{S}$ et al. Gastrointestinal motility during pregnancy: role of nitrergic component of NANC nerves. Am J Physiol Regul Integr Comp Physiol. 2000; 279: R1478-R1485

16. Chen $Q$ et al. Downregulation of Galphaq-11 protein expression in guinea pig antral and colonic circular muscle during pregnancy. Am J Physiol. 1999; 276: G895-G900

17. Bani D et al. Relaxin depresses small bowel motility through a nitric oxide-mediated mechanism. Studies in mice. Biol Reprod. 2002; 66: 778-84.

18. Zhou Q, O'Brien B, Relyea J. Severity of nausea and vomiting during pregnancy; what does it predict? Birth. 1999; 26:108-14. [PubMed: 10687575]

19. Macfie AG, Magides AD, Richmond MN, et al. Gastric emptying in pregnancy. Br J Anaesth.1991; 67:54-7. [PubMed: 1859760]

20. Jueckstock JK, Kaestner R, Mylonas I. Managing hyperemesis gravidarum: a multimodal challenge. BMC Medicine. 2010; 8:46. [PubMed: 20633258]

21. Hod M, Orvieto R, Kaplan B, et al. Hyperemesis gravidarum: a review. J Reprod Med. 1994;39: 605. [PubMed: 7996524]

22. Deuchar N. Nausea and vomiting in pregnancy: A review of the problem with particular regard to psychological and social aspects. Br J Obstet Gynaecol. 1995; 120:6-8. [PubMed: 7833313]
23. Davis M. Nausea and vomiting of pregnancy: an evidence-based review. J Perinatal Neonat Nurs.2004; 18:312-28.

24. Rosen T, Veciana M, Miler $\mathrm{H}$, et al. A randomized controlled trial of nerve stimulation for relief of nausea and vomiting in pregnancy. Obstet Gynecol. 2003; 102:129-35. [PubMed: 12850618]

25. Quinlan PD, Hill DA. Nausea and vomiting of pregnancy. American Family Physician. 2003; 68:121-28. [PubMed: 12887118]

26. American College of Obstreticians and Gynecologists. ACOG practice bulletin. Clinical management guidelines for obstretician-gynecologists: nausea and vomiting of pregnancy. Obstet Gynecol. 2004; 103:803-11. [PubMed: 15051578]

27. Ensiyeh J, Sakineh MA . Comparing ginger and vitamin B6 for the treatment of nausea vomiting in pregnancy: a randomised controlled trial. Midwifery. 2008 [doi:10.1016/j.midw.2007.10.013]

28. Mahady G, Pendland S, Y un G, et al. Ginger and the gingerols inhibits the growth of CagA+ strains of Helicobacter Pylori. Anticancer Res. 2003; 23:3699_ 3702. [PubMed: 14666666]

29. Vutyavanich T, Kraisarin T, Ruangsri RA. Ginger for nausea and vomiting in pregnancy: a randomized, doublemasked placebo-controlled trial. Obstet Gynecol. 2001; 97:577-82.[PubMed: 11275030]

30. Jarvis S. Management of nausea and vomiting in pregnancy. Br Med J. 2011;342:d3606

31. Festin M. Nausea and vomiting in early pregnancy. Clin Evid. 2009;6:1405.

32. Seto A, Einarson T, Koren G. Pregnancy outcomes following first trimester exposure to antihistamines: a meta-analysis. Am J Perinatol. 1997; 14:119-24.

[PubMed: 9259911]

33. Miklovich L, Van den Berg BJ. An evaluation of the teratogenicity of certain antinauseant drugs. Am J Obstet Gynecol. 1976; 125:244-48. [PubMed: 773181]

34. Koch KL, Frissora C. Nausea and vomiting during pregnancy. Gastroenterol Clin N Am. 2003; 32:201-34.

35. Mazzotta P, Magee LA. A risk-benefit assessment of pharmacological and non-pharmacological treatments for nausea and vomiting of pregnancy. Drugs. 2000; 50(4):781-800. 
36. Sorensen HT, Nielsen GL, Christensen K, TageJensen U, Ekbom A, Baron J. Birth outcome following maternal use of metoclopramide. Br J Clin Pharmacol. 2000;49:264-68.

37. Rodriguez-Pinilla E, Martinez-Frias ML. Corticosteroids during pregnancy and oral clefts: a casecontrol study. Teratology. 1998;58: 2-5

38. Gur C, Diav-Citrin O, Shechtman S, Arnon J, Ornoy A. Pregnancy outcome after first trimester exposure to corticosteroids: a prospective controlled study. Reprod Toxicol. 2004;18:93-101.

39. Rubenstein EB, Slusher BS, Rojas C, et al. New approaches to chemotherapy-induced nausea and vomiting: from neuropharmacology to clinical investigations. Cancer J. 2006; 12:341-47. [PubMed: 17034670]

40. Einarson A, Malatepe C, Navioz Y. The safety of ondansetron for nausea and vomiting of pregnancy: a prospective comparative study. Br J Obstet Gynecol. 2004; 111:940-43.

41. Ali RA, Egan LJ. Gastroesophageal reflux disease in pregnancy. Best Pract Res Clin Gastroenterol. 2007;21(5):793-806.

42. Richter JE. Gastroesophageal reflux disease during pregnancy. Gastroenterol Clin North Am. 2003;32:235-61.

43. VanThiel DH, Gavaler JS, Joshi SN, et al. Heartburn of pregnancy. Gastroenterology. 1977; 72: 668-78.

44. Mahadevan U, Kane S. American Gastroenterological Association Institute medical position statement on the use of gastrointestinal medications in pregnancy. Gastroenterology. 2006;131(1):278-82.

45. Tytgat GN, Heading RC, Muller-Lissner S, et al. Contemporary understanding and management of reflux and constipation in the general population and pregnancy: a consensus meeting. Aliment Pharmacol Ther. 2003; 18: 291-301.

46. Lindow SW, Regnell P, Sykes J, Little S. An openlabel multicenter study to assess the safety and efficacy of a novel reflux supplement (Gaviscon advance) in the treatment of heartburn of pregnancy. Int J Clin Pract. 2003; 57: 175-9.

47. Mahadevan U. Gastrointestinal medications in pregnancy. Best Pract Res Clin Gastroenterol. 2007;21(5):849-77.
48. Briggs GG, Freeman RY, Yaffe SJ. Drugs in Pregnancy and Lactation: A Reference Guide to Fetal and Neonatal Risk. Baltimore, USA: William and Wilkins, 2002.

49. Bailey B, Addis A, Lee A, et al. Cisapride use during human pregnancy. A prospective controlled multi-center study. Dig Dis Sci. 1997; 42: 1848-52.

50. Finkelstein W, Isselbacker KJ. Cimetidine. N Engl J Med. 1978; 229: 992-6.

51. Parker S, Schade RR, Pohl CR, et al. Prenatal and neonatal exposure of male pups to cimetidine but not ranitidine adversely affects subsequent adult sexual function. Gastroenterology. 1984; 86: 675-80.

52. Savarino V, Giasti M, Scalabrini P, et al. Famotidine has no significant effect on gonadal function in men. Gastroenterol Clin Biol. 1988; 12: 19-22.

53. Nikfar S, Abdollahi M, Moretti ME, et al. Use of proton pump inhibitors during pregnancy and rates of major malformations - A meta-analysis. Dig Dis Sci. 2002; 47: 1526-9.

54. Jewell DJ, Young G. Interventions for treating constipation in pregnancy. Cochrane Database Syst Rev. 2001;(2):CD001142.

55. Cullen G, O'Donoghue D. Constipation and pregnancy. Best Pract Res Clin Gastroenterol 2007;21(5):807-18.

56. Tack J, Müller-Lissner S, Stanghellini V, Boeckxstaens G, Kamm MA, Simren M, et al. Diagnosis and treatment of chronic constipation-a European perspective. Neurogastroenterol Motil. 2011; 23(8):697710.

57. Jick H, Holmes LB, Hunter JR, Madsen S, Stergachis A. First-trimester drug use and congenital disorders. JAMA. 1981;246(4):343-6.

58. Schindler AM. Isolated neonatal hypomagnesaemia associated with maternal overuse of stool softener. Lancet. 1984;2(8406):822.

59. Carulli N, Salvioli GF, Manenti F. Absorption of lactulose in man. Digestion. 1972;6(3):139-45.

Dr. A. V. Kishore Babu ${ }^{1}$, Dr. A. Srinivasa Rao ${ }^{2}$ ${ }^{1}$ Department of Pharmacy Practice, Bhaskar Pharmacy College, Moinabad, Hyderabad, Telangana, India. ${ }^{2}$ Department of Pharmacy Practice, Bhaskar Pharmacy College, Moinabad, Hyderabad, Telangana, India. 